\title{
Epidemiological analysis of multicentric and unicentric Castleman disease and TAFRO syndrome in Japan
}

\author{
Yasufumi Masaki, ${ }^{1)}$ Hiroshi Kawabata, ${ }^{1)}$ Shino Fujimoto, ${ }^{1)}$ Mitsuhiro Kawano, ${ }^{2)}$ Noriko Iwaki, \\ Takeharu Kotani, ${ }^{4)}$ Akikatsu Nakashima, ${ }^{5}$ Nozomu Kurose, ${ }^{6}$ Kazue Takai, ${ }^{7)}$ Ritsuro Suzuki, ${ }^{8)}$ \\ Sadao Aoki ${ }^{9)}$
}

Castleman disease is a polyclonal lymphoproliferative disease which is clinically classified into unicentric (UCD) and multicentric (MCD). TAFRO syndrome is a relatively new concept that partly overlaps with MCD. Due to their rarity, their incidence remains unknown. This study investigated the incidence and prevalence of UCD, MCD, and TAFRO syndrome in Japan using a fixed-point observation method based on their incidence in Ishikawa prefecture. The annual incidences of MCD, UCD, and TAFRO syndrome in Japan were 309-731, 71-542, and 110-502, respectively, yielding annual incidence rates per million individuals of 2.4-5.8, 0.6-4.3, and 0.9-4.9, respectively, and nationwide prevalence of 4,180-14,900, 1,350-10,300, and 860-7,240, respectively. In conclusion, MCD, UCD and TAFRO syndrome may not be as rare as previously estimated in Japan.

Key words: disease incidence, Statistical Research Committee of Japanese Society of Hematology, specific use survey, fixedpoint observation method

\section{INTRODUCTION}

Castleman disease was initially described as a lymphoproliferative disorder causing mediastinal masses characterized by abnormal histopathology. ${ }^{1}$ Castleman disease has since been subclassified clinically into unicentric (UCD) and multicentric Castleman disease (MCD), ${ }^{2}$ and subclassified histopathologically into the hyaline-vascular type (HV), plasma-cell type (PC), mixed type, plasmablastic type, and hypervascular type. ${ }^{3}$ MCD was recently subclassified into human herpes virus-8 (HHV-8)-associated, HHV-8unassociated (idiopathic MCD [iMCD]), POEMS syndromeassociated, and others. ${ }^{3}$

A new clinical entity, TAFRO syndrome, characterized by thrombocytopenia, anasarca (edema, pleural effusion, and ascites), fever, reticulin myelofibrosis (or renal insufficiency), and organomegaly (hepatosplenomegaly and lymphadenopathy), was recently described. ${ }^{4}$ Its diagnostic criteria and disease severity criteria, determined by the All Japan TAFRO
Syndrome Research Group in the Research Program for Intractable Diseases of the Ministry of Health, Labour and Welfare (MHLW) of Japan, ${ }^{5}$ were recently updated (https:// www.facebook.com/CastlemanTAFRO). As the lymph nodes of individuals with TAFRO syndrome were characterized histopathologically as Castleman disease, ${ }^{6}$ TAFRO syndrome has been categorized as part of iMCD.

Recently, clinical and basic studies on Castleman disease and TAFRO syndrome were started in Japan and the USA. We collected and analyzed data from more than 200 patients with iMCD, TAFRO syndrome, or conditions mimicking these disorders. Most patients with iMCD exhibit a chronic/ indolent clinical course, characterized by polyclonal hypergammopathy, multiple lymphadenopathy, and thrombocytosis. In contrast, most patients with TAFRO syndrome have an acute or sub-acute onset and progressive clinical course, characterized by normal to reduced gammaglobulin levels, thrombocytopenia, small or unnoticeable lymph nodes, and severe pleural effusion, ascites, and anasarca. Lymph node

\footnotetext{
Received: June 4, 2019. Revised: July 1, 2019. Accepted: July 16, 2019. J-STAGE Advance Published: November 8, 2019 DOI: $10.3960 /$ jslrt. 19021

${ }^{1)}$ Department of Hematology and Immunology, Kanazawa Medical University, Kahoku-gun, Ishikawa, Japan, ${ }^{2)}$ Division of Rheumatology, Department of Internal Medicine, Kanazawa University Hospital, Kanazawa, Japan, ${ }^{3)}$ Hematology/Respiratory Medicine, Faculty of Medicine, Institute of Medical, Pharmaceutical and Health Sciences, Kanazawa University, Kanazawa, Japan, ${ }^{4)}$ Division of Hematology, Ishikawa Prefectural Central Hospital, Kanazawa, Japan, ${ }^{5}$ Division of Nephrology and Rheumatology, Ishikawa Prefectural Central Hospital, Kanazawa, Japan, ${ }^{6}$ Department of Pathology and Laboratory Medicine, Kanazawa Medical University, Kahoku-gun, Ishikawa, Japan, ${ }^{7)}$ Department of Hematology, Niigata City General Hospital, Niigata, Japan, ${ }^{8)}$ Shimane University Hospital Innovative Cancer Center, Izumo, Japan, ${ }^{9)}$ Division of Pathophysiology, Faculty of Pharmaceutical Sciences, Niigata University of Pharmacy and Applied Life Sciences, Niigata, Japan

Corresponding author: Yasufumi Masaki; Department of Hematology and Immunology, Kanazawa Medical University, 1-1 Daigaku, Uchinada, Kahoku-gun, Ishikawa 920-0293, Japan. E-mail: yasum@kanazawa-med.ac.jp 
biopsy specimens cannot be obtained from some patients with TAFRO syndrome because of marked bleeding tendency, anasarca, and/or the absence of apparent lymphadenopathy. Although some histological findings may overlap, we speculate that TAFRO syndrome is a distinct clinical entity from typical iMCD.

As there is little epidemiological information on Castleman disease and TAFRO syndrome in Japan, we assumed the epidemiology of these conditions using a fixedpoint observation method based on their incidence in Ishikawa prefecture.

\section{PATIENTS AND METHODS}

\section{Disease classification}

The diagnosis of Castleman disease is based on both tentative diagnostic criteria in $\mathrm{Japan}^{7}$ and consensus diagnostic criteria for HHV-8-negative/idiopathic MCD in the USA. ${ }^{3}$ UCD is defined by the involvement of a single lymph node or by the restriction of affected lymph nodes to a single site, enabling surgical resection. MCD is defined by the involvement of lymph nodes at multiple sites.

The diagnostic criteria for TAFRO syndrome have been previously described. ${ }^{5}$ Patients with TAFRO syndrome manifesting lymphadenopathy with a Castleman-disease like histology (TAFRO-iMCD) were classified into TAFRO syndrome but not into iMCD.

\section{Epidemiological analysis}

The incidence of MCD, UCD, and TAFRO syndrome throughout Japan was estimated by a fixed-point observation method based on their incidences in Ishikawa prefecture. This prefecture is located on the coast of the Sea of Japan on the Honshu island, and is relatively rural with few of its $1,140,000$ residents moving in or out of this area. This study assumed that most patients in Ishikawa prefecture who developed MCD, UCD, or TAFRO syndrome were seen at 3 major medical centers, i.e., Kanazawa University Hospital, Ishikawa Prefectural Central Hospital, or Kanazawa Medical University Hospital. Patient data were collected from medical records, and this study was approved by the ethics committees of each institute.

The rates of MCD, UCD, and TAFRO syndrome at these three hospitals were discussed among the authors of this paper, and the hematologists and rheumatologists working there, and were estimated to be $60-90 \%, 35-65 \%$, and $35-65 \%$, respectively. The 10 -year survival rates of patients with MCD and UCD were estimated to be $80 \%{ }^{8}$ and $95 \%,{ }^{4}$ respectively, and the 5-year survival rate of patients with TAFRO syndrome was estimated to be $67 \%$. The total numbers of patients with MCD, UCD, or TAFRO syndrome diagnosed at these three hospitals from 2012 through 2018 were collected, and the incidence and prevalence of these conditions were calculated using a fixed-point observation method.

\section{RESULTS}

The numbers of diagnosed cases of MCD, UCD, and TAFRO syndrome at the 3 major medical centers in Ishikawa Prefecture were 23, 9, and 8, respectively. In 8 cases of TAFRO syndrome, 7 were TAFRO-iMCD and one lacked lymphadenopathy.

Assuming that the onset follows a Poisson distribution, the annual numbers of individuals in Japan with MCD, UCD, and TAFRO syndrome in Japan were estimated to be 309$731,71-542$, and 110-502, respectively, yielding annual incidence rates per million individuals of 2.4-5.8, 0.6-4.3, and $0.9-4.9$, respectively. The prevalence of MCD, UCD, and TAFRO syndrome in Japan was estimated to be 4,180 14,900, 1,350-10,300, and 860-7,240, respectively.

\section{DISCUSSION}

Castleman disease can affect patients over a broad age range, from children to the elderly, with median ages of onset being in the 50's for patients with MCD and in the 30's for patients with UCD. ${ }^{8}$ UCD is reported to be more common in Western countries, China, and South Korea, whereas MCD is more common in Japan. ${ }^{8-10}$ The Japanese Society of Hematology case registration system of hematological disorders reported that $70 \%$ of patients with Castleman disease have MCD, whereas 30\% have UCD. ${ }^{11}$ Most patients with HHV-8-associated MCD are infected with human immunodeficiency virus (HIV). ${ }^{12}$ More than $80 \%$ of MCD patients in a study from France were HHV-8-positive. ${ }^{13}$ In contrast, HHV-8-associated MCD is markedly rare in Japan. ${ }^{14,15}$

Based on data from two US treatment centers, the Fred Hutchinson Cancer Research Center and the Mayo Clinic, the prevalence of Castleman disease in the USA is 0.65-2.37 per million persons. ${ }^{16}$ Furthermore, a claims database estimated that the incidence of CD was 21-25 per million people, with $23 \%$ of these patients thought to have MCD. ${ }^{17}$ However the reliability of these surveys is questionable due to the 10 -fold difference between them.

The Research Group on Castleman Disease formed by the Ministry of Health, Labour and Welfare, Japan estimated that the annual incidence of MCD in Japan is one per 1 million people (120 per year) and the prevalence is 1,500 patients (http:// www.nanbyou.or.jp/entry/5750) based on data of a multicenter collaborative retrospective study to establish the concept of TAFRO syndrome (UMIN000011809), and by the data of a specific-use survey of tocilizumab $\left(\right.$ Actemra $\left.^{R}\right)$ by Chugai Pharmaceutical Co., Ltd. (http://chugai-pharm.jp/hc/ss/pr/drug/ act_via0400/report/scs/PDF/20140225_act_epmpv_safe_cd.pdf).

Over the last decade from $200 \overline{8}$ to 2017 , the Statistical Research Committee of the Japanese Society of Hematology has registered 320 cases of MCD, 142 of UCD, and 57 of unclassified Castleman disease. These numbers slightly increased over time (Figure 1). In 2017, 73 cases of MCD, 28 of UCD, and two of unclassified Castleman disease were registered. If the capture rate of MCD and UCD in 2017 in this registration period was assumed to be $20-40 \%$ and 


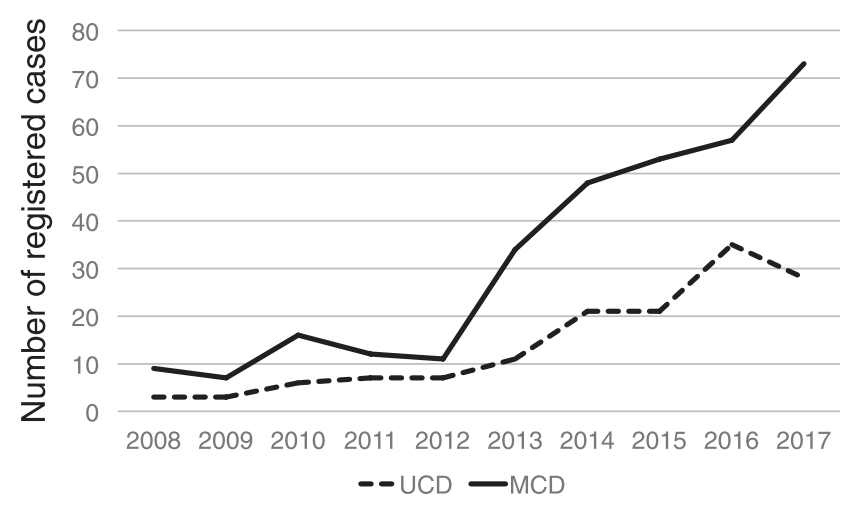

Fig. 1. Annual number of MCD and UCD cases registered by the Statistical Research Committee of the Japanese Society of Hematology. The incidence of these diseases has increased over time.

$15-30 \%$, respectively, the annual incidences in Japan would be 183-365 and 83-167, respectively. There was no data for TAFRO syndrome in this survey.

Compared with these prior estimations in Japan, this study using the fixed-point observation method, revealed an at least 2-fold increase in the annual incidence and a 4-fold increase in the prevalence of MCD in Japan. The annual incidences of UCD and MCD estimated by the fixed-point observation method were largely consistent with the estimation using the data of the latest year in the nation-wide registration study conducted by the Japanese Society of Hematology. The number of registered patients with Castleman disease in the registry has slightly increased over time, possibly due to increases in the capture rates rather than increases in the actual incidence. In April 2018, the Ministry of Health, Labour and Welfare included iMCD as one of the designated intractable diseases that may have led to the increase in registered cases by altering its recognition. We also estimated the annual incidence of TAFRO syndrome to be 110-502, which is higher than previously thought.

Although Castleman disease has a long history, it is rare and difficult to diagnose, resulting in underestimation of its incidence and prevalence. Moreover, due to insufficient awareness of TAFRO syndrome, some case may have been misdiagnosed as other diseases. ${ }^{4}$ The present study suggested that MCD, UCD, and TAFRO syndrome are not as rare as previously assumed in Japan. However, these results are limited in that they were derived from regional data. As the etiologies of these diseases are not fully understood and therapeutic strategies have not been established, especially for TAFRO syndrome, further nationwide clinical and epidemiological studies are warranted.

\section{GRANTS SUPPORT}

This study was supported in part by grants from the Ministry of Health, Labour and Welfare, Japan (H29 Nanchi, etc. (Nan)-General-019, H27-28 Nanchi, etc. (Nan)General-002 and -008), and by the Japanese Ministry of Education, Culture, Sports, Science and Technology (Grant
No. 17591060 and 15K09510), the Kanazawa Medical University Research Foundation (Grant Nos. S2004-16 and S2007-5), Grant for Assist KAKEN from Kanazawa Medical University (Grant No. K2011-7), Grant for Project Research from High-Tech Research Center of Kanazawa Medical University (Grant No. H2011-11), and Grant for Alumni Research (A) from Kanazawa Medical University (AR2012-06).

\section{ACKNOWLEDGMENTS}

We thank all participants of the All Japan, Ministry of Health, Labour, and Welfare (MHLW) TAFRO syndrome Team and Castleman disease Team for their help and critical discussion.

\section{CONFLICTS OF INTEREST}

YM received research grants from Kyowa Hakko Kirin Pharma, Astellas Pharma, Eisai Pharma, Ono Pharma, Pfizer Pharma, Asahi Kasei Pharma, MSD Pharma, Daiichi-Sankyo Pharma, Taisho Pharma, Taiho Pharma, Takeda Pharma, Chugai Pharma, Teijin Pharma, Nippon Kayaku, and Mochida Pharma outside the submitted work. RS received honoraria from Kyowa Hakko Kirin. All of the other authors have no conflicts of interest.

\section{REFERENCES}

1 Castleman B, Iverson L, Menendez VP. Localized mediastinal lymph-node hyperplasia resembling thymoma. Cancer. 1956; 9 : 822-830.

2 Frizzera G. Castleman's disease and related disorders. Semin Diagn Pathol. 1988; 5 : 346-364.

3 Fajgenbaum DC, Uldrick TS, Bagg A, et al. International, evidence-based consensus diagnostic criteria for HHV-8-negative/ idiopathic multicentric Castleman disease. Blood. 2017; 129 : 1646-1657.

4 Takai K, Nikkuni K, Shibuya H, Hashidate H. [Thrombocytopenia with mild bone marrow fibrosis accompanied by fever, pleural effusion, ascites and hepatosplenomegaly]. Rinsho Ketsueki. 2010; $51: 320-325$ [in Japanese].

5 Masaki Y, Kawabata H, Takai K, et al. Proposed diagnostic criteria, disease severity classification and treatment strategy for TAFRO syndrome, 2015 version. Int J Hematol. 2016; 103 : 686-692.

6 Kurose N, Futatsuya C, Mizutani K, et al. The clinicopathological comparison among nodal cases of idiopathic multicentric Castleman disease with and without TAFRO syndrome. Hum Pathol. 2018; 77 : 130-138.

7 Fujimoto S, Koga T, Kawakami A, et al. Tentative diagnostic criteria and disease severity classification for Castleman disease: A report of the research group on Castleman disease in Japan. Mod Rheumatol. 2018; 28 : 161-167.

8 Ye B, Gao SG, Li W, et al. A retrospective study of unicentric and multicentric Castleman's disease: a report of 52 patients. Med Oncol. 2010; 27 : 1171-1178. 
9 Talat N, Schulte KM. Castleman's disease: systematic analysis of 416 patients from the literature. Oncologist. 2011; 16 : 1316-1324.

10 Shin DY, Jeon YK, Hong YS, et al. Clinical dissection of multicentric Castleman disease. Leuk Lymphoma. 2011; 52 : 1517-1522.

11 Nakao S. [Recent trend in the incidence of hematologic disease in Japan: a report from Statistical Research Comittee of Japanese Society of Hematology]. Rinsho Ketsueki. 2013; 54 : 2079-2085 [in Japanese].

12 Lowenthal DA, Filippa DA, Richardson ME, Bertoni M, Straus DJ. Generalized lymphadenopathy with morphologic features of Castleman's disease in an HIV-positive man. Cancer. 1987; 60 : 2454-2458.

13 Oksenhendler E, Boutboul D, Fajgenbaum D, et al. The full spectrum of Castleman disease: 273 patients studied over 20 years. Br J Haematol. 2018; 180 : 206-216.
14 Kojima M, Nakamura N, Tsukamoto N, et al. Clinical implications of idiopathic multicentric Castleman disease among Japanese: a report of 28 cases. Int J Surg Pathol. 2008; 16 : 391-398.

15 Kawabata H, Kadowaki N, Nishikori M, et al. Clinical features and treatment of multicentric Castleman's disease : A retrospective study of 21 Japanese patients at a single institute. J Clin Exp Hematop. 2013; 53 : 69-77.

16 Robinson D Jr, Reynolds M, Casper C, et al. Clinical epidemiology and treatment patterns of patients with multicentric Castleman disease: results from two US treatment centres. Br J Haematol. 2014; 165 : 39-48.

17 Munshi N, Mehra M, van de Velde H, et al. Use of a claims database to characterize and estimate the incidence rate for Castleman disease. Leuk Lymphoma. 2015; 56 : 1252-1260. 